\title{
Analytic study on backreacting holographic superconductors with dark matter sector
}

\author{
Łukasz Nakonieczny and Marek Rogatkd* \\ Institute of Physics \\ Maria Curie-Sklodowska University \\ 20-031 Lublin, pl. Marii Curie-Sklodowskiej 1, Poland
}

(Dated: March 7, 2018)

\begin{abstract}
The variational method for Sturm-Liouville eigenvalue problem was employed to study analytically properties of the holographic superconductor with dark matter sector, in which a coupling between Maxwell field and another $U(1)$-gauge field was considered. The backreation of the dark matter sector on gravitational background in question was also examined.

PACS numbers: $11.25 . \mathrm{Tq}, 04.50 .-\mathrm{h}, 98.80 . \mathrm{Cq}$
\end{abstract}

\section{INTRODUCTION}

The AdS/CFT correspondence [1] plays a significant role in understanding the strongly coupled field theories. It relates string theory on asymptotically anti-de Sitter spacetime (AdS) to a conformal field theory on the boundary and enables to establish method for obtaining correlation functions in a strongly interacting field theory by means of a dual classical gravity description [2]. The aforementioned correspondence provides a theoretical insight into description of Hall and Nernst effects [3, 4] (see also some contemporary reviews and references therein [5]). Moreover, it has been also successful applied to nuclear physics problems like heavy ion collisions at the Relativistic Heavy Ion Collider (RHIC) [6].

It has been also proposed that AdS/CFT correspondence could be useful in describing superconductor phase transitions, namely when an Abelian symmetry is broken outside an AdS black hole this phenomenon results in the formation of the holographic superconductor (formation of charged scalar condensation) in the dual CFT [7]-[8]. It was indicated that the expectation value of charged operators underwent the $U(1)$-symmetry breaking and the second order phase transition took place [9]-[11]. Other properties of superconducting materials bounded with the influence of the magnetic field, were also reconstructed. The aforementioned studies covered the topic of critical magnetic fields and Abrikosov vortices [12]-[13]. At the beginning, studies of the holographic superconductivity when no backreations of matter fields were considered. Contrary to the previously elaborated cases, the backreaction caused that even uncharged scalar fields could form a condensation in $(2+1)$-dimensions [11]. In the case of p-wave holographic models it was shown that the phase transition leading to the formation of vector hair, changed from the second order to the first one, depending on the strength of the gravitational coupling [14]-15]. These facts have triggered studies of the holographic superconductivity including the backreaction effect of the matter fields in question (see, e.g., Refs. [16]-[17]). Recently, also the backreaction between holographic insulator and superconductor was considered [18]-21.

The other theories of gravity were also taken into account. Holographic superconductivity in Einstein-GaussBonnet (EGB) gravity were elaborated and among all it was revealed that the higher curvature corrections made the condensations harder to occur [22]-26]. Realization of holographic superconductivity in Einstein-Maxwell-dilaton gravity being a generalized model built from the most extensive covariant gravity Lagrangian with at most two derivatives of fields 27] was given in Ref.[28]. On the other hand, gravity theory including dilaton field were also investigated in the case of the potential models of superfluids and superconductors [29]. Horava-Lifshitz gravity [30] and string/M theory [31] were also paid attention to. Additionally other kind of electro-magnetic theories were studied due to the holographic superconductivity (see for example [32] and references therein). For instance, the presence of the Born-Infeld scale parameter decreases the critical temperature and the ratio of the gap frequency in conductivity, comparing to the Maxwell electrodynamics. Recently, also frameworks of non-linear electrodynamics acquire much attention due to holographic superconductivity studies [33]. On the other hand, analytical methods of investigations of the holographic superconductivity with or without a backreaction were examined in Refs. [34] (see e.g., for the selected examples of this still growing literature).

Furthermore, it naturally arises a question about possible matter configurations in AdS spacetime. In Ref. 35] it was revealed that strictly stationary Einstein-Maxwell spacetime with negative cosmological constant could not allow for

*Electronic address: rogat@kft.umcs.lublin.pl, marek.rogatko@poczta.umcs.lublin.pl, lnakonieczny@kft.umcs.lublin.pl 
the existence of nontrivial configurations of complex scalar fields or form fields. This statement was also confirmed in the case of strictly stationary, simply connected Einstein-Maxwell-axion-dilaton spacetime with negative cosmological constant and arbitrary number of $U(1)$-gauge fields [36].

Present observations of the Universe envisage the fact that it may be dominated by an exotic kind of fields never observed directly. In the late 1990s the independent surveys for distant supernovae type Ia (Sn Ia), calibrated as standard candles, announced that the high-redshifted supernovae of this type appeared about 40 percent fainter or equivalently more distant than expected in a flat, matter-dominated Universe [37]. Other investigations like analysis of cosmic microwave background [38] or baryonic acoustic oscillations [39] confirm that our Universe is filled with the exotic matter with negative pressure, causing its acceleration.

The contemporary observations provide also the strong evidence that almost 22 percent of the total energy density of our Universe is of a form of dark matter, which on its turn is unclear what it is made of. Recently, the new model mimicking the dark matter sector was proposed (see e.g., for the latest issues Refs. [40]). In the aforementioned prediction the standard model is coupled to the dark sector via an interactive term. It has the form of a direct coupling between $U(1)$-gauge strength tensor of Maxwell field and the dark matter one. It worth pointing out that, the interaction of $U(1)$ Abelian-Higgs field model having cosmic string solutions with a low energy dark matter sector being also a $U(1)$ Abelian-Higgs model was studied in the context of cosmic string interacting with dark string [41].

In order to generalize existing results concerning holographic superconductors as well as motivated by the above astrophysical observations, we shall study the influence of the proposed dark matter sector coupled to the standard model on holographic superconductivity. We shall consider bottom up theory and study backreaction of the considered matter fields on gravitational background in question.

The paper is organized as follows. In Sec.II we derive equations of motion for a holographic superconductor model with backreactions in n-dimensional AdS black hole background, in the underlying theory. Sec.III is devoted to the analytical description of superconducting phase, while in Sec.IV the critical temperature dependence on the spacetime dimension as well as parameters characterizing dark matter sector are examined. In Sec.V we present remarks and conclusions of our investigations.

\section{ACTION AND EQUATIONS OF MOTION}

In this section one considers a holographic Abelian-Higgs model for s-wave superconductor. In the matter sector we assume a coupling between Maxwell gauge field and another $U(1)$-gauge field in order to represent the dark matter influence. We remark that two gauge uncoupled fields were studied in the case of the so-called unbalanced holographic superconductor [46]. On the other hand, holographic Abelian-Higgs model for s-wave superconductivity with a coupling between the gauge field and a new massive gauge field introduced in order to describe impurities was examined in Refs. [47]. In what follows we shall for the first time study backreaction of dark matter sector on the gravitational background. Perhaps considering the phase transition temperature enables one to find justification and evidence of the aforementioned dark matter model in the future experiments.

To commence with we shall specify the gravitational part of the action which implies

$$
S_{g}=\int \sqrt{-g} d^{n} x \frac{1}{2 \kappa^{2}}(R-2 \Lambda)
$$

where $\kappa^{2}=8 \pi G_{n}$ is an n-dimensional gravitational constant. The cosmological constant will be given by $\Lambda=$ $-\frac{(n-1)(n-2)}{2 L^{2}}$, where $L$ is the radius of the AdS spacetime. The underlying matter sector is modelled by the AbelianHiggs sector coupled to the second $U(1)$-gauge field. It is provided by the following action:

$$
S_{m}=\int \sqrt{-g} d^{n} x\left(-\frac{1}{4} F_{\mu \nu} F^{\mu \nu}-\left[\nabla_{\mu} \psi-i q A_{\mu} \psi\right]^{\dagger}\left[\nabla^{\mu} \psi-i q A^{\mu} \psi\right]-V(\psi)-\frac{1}{4} B_{\mu \nu} B^{\mu \nu}-\frac{\alpha}{4} F_{\mu \nu} B^{\mu \nu}\right),
$$

where the scalar field potential satisfies $V(\psi)=m^{2}|\psi|^{2}+\frac{\lambda_{\psi}}{4}|\psi|^{4} . \quad F_{\mu \nu}=2 \nabla_{[\mu} A_{\nu]}$ stands for the ordinary Maxwell field strength tensor, while the second $U(1)$-gauge field $B_{\mu \nu}$ is given by $B_{\mu \nu}=2 \nabla_{[\mu} B_{\nu]}$. Moreover, $m, \lambda_{\psi}, q$ represent mass, a coupling constant and charge related to the scalar field $\psi$, respectively. On the other hand, $\alpha$ is a coupling constant for both $U(1)$ fields. In order to be compatible with the current observations it should be on the order of $10^{-3}$.

The motivation standing behind our studies is related to the fact that one has the strong evidence that almost 22 percent of the total energy density of our Universe is in the form of dark matter. Up till now we have only vague idea what it is made of. The model in which dark matter sector is coupled to the Standard Model has its possible roots in astrophysical observations of $511 \mathrm{keV}$ gamma rays observed by Integral/SPI [42] and experiments revealing 
electron and positron excess observed in galaxy by ATIC 43] and PAMELA [44]. It turned out that the energy of the aforementioned excess is in the range between a few of $\mathrm{GeV}$ to a few of $\mathrm{TeV}$. The conceivable explanation is the annihilation of dark matter into electrons, i.e., below $\mathrm{GeV}$ scale, the interaction term can be in the form of a direct coupling between $U(1)$-gauge strength tensor of the dark matter sector and $U(1)$ Maxwell strength tensor.

There are also other possible evidences revealing new physics, e.g., the $3.6-\sigma$ discrepancy between measured value of the muon anomalous magnetic moment and its prediction in the Standard Model [45]. From the cosmological point of view, these kinds of dark matter models admitted cosmic string like solutions, topological defects which might arise in the early Universe [41]. In the above context it will be interesting to look for the imprints of dark matter sector in properties of holographic superconductors and reveal the other experimental confirmation of the underlying model.

The Einstein equations for the system in question may be written in the form as

$$
R_{\mu \nu}=\left(\frac{n}{n-2}-1\right) \Lambda g_{\mu \nu}+2 \kappa^{2}\left(T_{\mu \nu}-\frac{1}{n-2} T\right)
$$

where the energy-momentum tensor for the matter fields yields

$$
\begin{aligned}
T_{\nu}^{\mu} & =F_{\alpha}^{\mu} F_{\nu}^{\alpha}+B_{\alpha}^{\mu} B_{\nu}^{\alpha}+\alpha B_{\alpha}^{\mu} F_{\nu}^{\alpha}+2 \nabla^{\mu} \psi \nabla_{\nu} \psi+2 q^{2} A^{\mu} A_{\nu} \psi^{2}+ \\
& +g_{\nu}^{\mu}\left(-\frac{1}{4} F_{\alpha \beta} F^{\alpha \beta}-\frac{1}{4} B_{\alpha \beta} B^{\alpha \beta}-\frac{\alpha}{4} F_{\alpha \beta} B^{\alpha \beta}-\nabla_{\alpha} \psi \nabla^{\alpha} \psi-q^{2} A_{\alpha} A^{\alpha} \psi^{2}-V(\psi)\right) .
\end{aligned}
$$

Variations of the underlying action with respect to the matter fields reveal the exact form of the equations of motion for the considered theory. They have the forms as follows:

$$
\begin{aligned}
& \nabla_{\mu} F^{\mu \nu}-2 q^{2} A^{\nu} \psi^{2}+\frac{\alpha}{2} \nabla_{\mu} B^{\mu \nu}=0 \\
& \nabla_{\mu} \nabla^{\mu} \psi-q^{2} A_{\mu} A^{\mu} \psi-\frac{1}{2} \frac{\partial V(\psi)}{\partial \psi}=0 \\
& \nabla_{\mu} B^{\mu \nu}+\frac{\alpha}{2} \nabla_{\mu} F^{\mu \nu}=0 .
\end{aligned}
$$

Having in mind the form of the relation (7) we see that the dynamics of the $B_{\mu}$ field is determined (up to the integration constant) by the Maxwell field potential $A_{\mu}$. After inserting equation (7) into the relation (5) we arrive at the following:

$$
\left(1-\frac{\alpha^{2}}{4}\right) \nabla_{\mu} F^{\mu \nu}-q^{2} \psi^{2} A^{\nu}=0
$$

In order to proceed further one introduces a general line element which enables us to consider backreation of matter fields on the gravitational sector. Namely, it is provided by

$$
d s^{2}=-f(r) e^{-\chi(r)} d t^{2}+\frac{d r^{2}}{f(r)}+r^{2} h_{i j} d x^{i} d x^{j}
$$

where $f$ and $\chi$ are functions of $r$-coordinate, $h_{i j}$ is the metric tensor on the $(n-2)$-dimensional submanifold. Further, we assume that the considered gauge fields posses only the temporal components which also depend only on the radial coordinate $r$. They can be written as

$$
A_{t}=\phi(r), \quad B_{t}=\eta(r)
$$

Consequently it leads to the equations of motion as follows:

$$
\begin{aligned}
& \partial_{r}^{2} \psi+\left(\frac{n-2}{r}+\frac{1}{f} \partial_{r} f-\frac{1}{2} \partial_{r} \chi\right) \partial_{r} \psi+\frac{e^{\chi} q^{2} \phi^{2}}{f^{2}} \psi-\frac{1}{2 f} \frac{\partial V}{\partial \psi}=0 \\
& \partial_{r}^{2} \phi+\left(\frac{1}{2} \partial_{r} \chi+\frac{n-2}{r}\right) \partial_{r} \phi-\frac{2 q^{2} \psi^{2}}{\tilde{\alpha} f} \phi=0 \\
& \partial_{r} \eta=-c_{1} r^{-n+2} e^{-\frac{1}{2} \chi}-\frac{\alpha}{2} \partial_{r} \phi
\end{aligned}
$$

where we have introduced $\tilde{\alpha}=1-\frac{\alpha^{2}}{4}$ and the integration constant $c_{1}$. 
On the other hand, the Ricci curvature tensor components yield

$$
\begin{aligned}
& R_{t}^{t}-R_{r}^{r}=\frac{n-2}{2 r} f \partial_{r} \chi, \\
& R_{x}^{x}=-\frac{1}{r} \partial_{r} f+\frac{1}{2 r} f \partial_{r} \chi-\frac{n-3}{r^{2}} f .
\end{aligned}
$$

One assumes that $(n-2)$-dimensional submanifold is flat and $x$ in $R_{x}^{x}$ represent the generic coordinate in this submanifold.

By virtue of the above relations one can readily verify that the complete set of the differential equations describing the system in question is determined by

$$
\begin{aligned}
\partial_{r} \chi & =-\frac{4 \kappa^{2} r}{n-2}\left(2\left(\partial_{r} \psi\right)^{2}+2 \frac{e^{\chi} q^{2} \phi^{2} \psi^{2}}{f^{2}}\right) \\
\partial_{r} f & +\frac{n-3}{r} f+\frac{2 r}{n-2} \Lambda=-\frac{2 \kappa^{2} r}{n-2}\left[2 f\left(\partial_{r} \psi\right)^{2}+2 \frac{e^{\chi} q^{2} \phi^{2} \psi^{2}}{f}\right. \\
& \left.+2 V(\psi)+e^{\chi}\left(\left(\partial_{r} \phi\right)^{2}+\left(\partial_{r} \eta\right)^{2}+\alpha \partial_{r} \phi \partial_{r} \eta\right)\right] \\
\partial_{r}^{2} \psi & +\left(\frac{n-2}{r}+\frac{1}{f} \partial_{r} f-\frac{1}{2} \partial_{r} \chi\right) \partial_{r} \psi+\frac{e^{\chi} q^{2} \phi^{2}}{f^{2}} \psi-\frac{1}{2 f} \frac{\partial V}{\partial \psi}=0 \\
\partial_{r}^{2} \phi & +\left(\frac{1}{2} \partial_{r} \chi+\frac{n-2}{r}\right) \partial_{r} \phi-\frac{2 q^{2} \psi^{2}}{\tilde{\alpha} f} \phi=0 \\
\partial_{r} \eta & =-c_{1} r^{-n+2} e^{-\frac{1}{2} \chi}-\frac{\alpha}{2} \partial_{r} \phi .
\end{aligned}
$$

Using equation (20) one can rewrite relation (17). Namely, $\eta$ field can be eliminated from the set of the differential equations system, and the modified relation (17) may be cast in the form as

$$
\partial_{r} f+\frac{n-3}{r} f+\frac{2 r}{n-2} \Lambda=-\frac{2 \kappa^{2} r}{n-2}\left[2 f\left(\partial_{r} \psi\right)^{2}+2 \frac{e^{\chi} q^{2} \phi^{2} \psi^{2}}{f}+2 V(\psi)+e^{\chi}\left(\tilde{\alpha}\left(\partial_{r} \phi\right)^{2}+a^{2}\right)\right],
$$

where we set $a^{2}=c_{1}^{2} r^{-2 n+4} e^{-\chi}$.

In the normal phase one has that $\psi(r)=0$, which in turn implies that $\chi$ is constant. Having this in mind the remaining equations may be rewritten in the forms as

$$
\begin{aligned}
& \partial_{r} f+\frac{n-3}{r} f+\frac{2 r}{n-2} \Lambda=-\frac{2 \kappa^{2} r}{n-2} e^{\chi}\left(\tilde{\alpha}\left(\partial_{r} \phi\right)^{2}+a^{2}\right), \\
& \partial_{r}^{2} \phi+\frac{n-2}{r} \partial_{r} \phi=0 .
\end{aligned}
$$

It is easy to verify that the solution of (23) yields

$$
\phi=\mu-\frac{\rho}{r^{n-3}},
$$

where $\mu$ and $\rho$ are interpreted as the chemical potential and charge density in the dual field theory. On the other hand, for $f(r)$ one arrives at the expression

$$
f=\frac{r^{2}}{L^{2}}+\frac{2 \kappa^{2}}{n-2}\left[\tilde{\alpha}(n-3) \rho^{2}+\frac{c_{1}^{2}}{n-3}\right] \frac{1}{r^{2 n-6}}-\left[\frac{r_{+}^{n-1}}{L^{2}}+\frac{2 \kappa^{2}}{n-2} \frac{\tilde{\alpha}(n-3) \rho^{2}+\frac{c_{1}^{2}}{n-3}}{r_{+}^{n-3}}\right] \frac{1}{r^{n-3}} .
$$

It can be observed that in the case when $\psi=0$ the line element under consideration represents the Reissner-NordströmAdS (RN-AdS) black hole.

In the next step we shall tackle the problem when $\psi \neq 0$. Namely, we pay attention to the appropriate boundary conditions. From the previous relations one has that the functions $\chi$ and $f$ satisfy at the black hole event horizon $r_{+}$ the following relations:

$$
\begin{aligned}
& \left(\partial_{r} \chi\right)_{\mid r_{+}}=-\frac{4 \kappa^{2} r_{+}}{n-2}\left[2\left(\partial_{r} \psi\right)_{\mid r_{+}}^{2}+2 \frac{e^{\chi\left(r_{+}\right)} q^{2}\left(\partial_{r} \phi\right)_{\mid r_{+}}^{2} \psi\left(r_{+}\right)^{2}}{\left(\partial_{r} f\right)_{\mid r_{+}}^{2}}\right], \\
& \left(\partial_{r} f\right)_{\mid r_{+}}=-\frac{2 r_{+}}{n-2} \Lambda-\frac{2 \kappa^{2} r_{+}}{n-2}\left[2 V\left(\psi\left(r_{+}\right)\right)+e^{\chi\left(r_{+}\right)}\left(\tilde{\alpha}\left(\partial_{r} \phi\right)_{\mid r_{+}}^{2}+a\left(r_{+}\right)^{2}\right)\right] .
\end{aligned}
$$


On the other hand, at the black hole event horizon, one finds that the boundary conditions yield

$$
\begin{aligned}
\phi\left(r_{+}\right) & =0 \\
\left(\partial_{r} f\right)_{\mid r_{+}}\left(\partial_{r} \psi\right)_{\mid r_{+}} & -\frac{1}{2}\left(\frac{\partial V}{\partial \psi}\right)_{\mid r_{+}}=0 \\
\left(\partial_{r} \eta\right)_{\mid r_{+}} & =-c_{1} r_{+}^{-n+2} e^{-\chi\left(r_{+}\right)}-\frac{\alpha}{2}\left(\partial_{r} \phi\right)_{\mid r_{+}}
\end{aligned}
$$

Next, it remains to check the behaviour of the above functions at the AdS boundary (when $r \rightarrow \infty$ ). Their asymptotical forms are provided by the following:

$$
\chi \rightarrow 0, \quad f \sim \frac{r^{2}}{L^{2}}, \quad \phi \sim \mu-\frac{\rho}{r^{n-3}}, \quad \psi \sim \frac{\psi_{-}}{r^{\Delta_{-}}}+\frac{\psi_{+}}{r^{\Delta_{+}}}, \quad \partial_{r} \eta=-c_{1} r^{-n+2}-\frac{\alpha}{2} \partial_{r} \phi,
$$

where the exponent is defined by the relation $\Delta_{ \pm}=\frac{1}{2}\left[n-1 \pm \sqrt{(n-1)^{2}+4 m^{2}}\right]$.

\section{ANALYTICAL DESCRIPTION OF SUPERCONDUCTING PHASE}

In this section we shall implement Sturm-Liouville method [51] to the analysis of behaviour of s-wave holographic superconductor phase transition with backreaction on the gravitational sector of the underlying theory. To proceed further, one introduces new variable $z=\frac{r_{+}}{r}$ and rewrite the equations of motion in the forms as

$$
\begin{aligned}
& \partial_{z} f-\frac{n-3}{z} f+\frac{(n-1) r_{+}^{2}}{L^{2} z^{3}}-\frac{1}{z^{3}} \frac{2 \kappa^{2} r_{+}^{2}}{n-2}\left(2 f\left(\frac{z^{4}}{r_{+}^{2}}\left(\partial_{z} \psi\right)^{2}+\frac{e^{\chi} q^{2} \phi^{2} \psi^{2}}{f^{2}}\right)\right. \\
& \left.+2 V(\psi)+e^{\chi}\left(\tilde{\alpha} \frac{z^{4}}{r_{+}^{2}}\left(\partial_{z} \phi\right)^{2}+\frac{c_{1}^{2}}{r_{+}^{2 n-4}} z^{2 n-4}\right)\right)=0 \\
& \partial_{z} \chi-\frac{1}{z^{3}} \frac{4 \kappa^{2} r_{+}^{2}}{n-2}\left(\frac{2 z^{4}}{r_{+}^{2}}\left(\partial_{z} \psi\right)^{2}+2 \frac{e^{\chi} q^{2} \phi^{2} \psi^{2}}{f^{2}}\right)=0, \\
& \partial_{z}^{2} \phi-\left(\frac{n-4}{z}-\frac{1}{2} \partial_{z} \chi\right) \partial_{z} \phi+\frac{2 r_{+}^{2}}{\tilde{\alpha} z^{4}} \frac{q^{2} \psi^{2}}{f} \phi=0 \\
& \partial_{z}^{2} \psi-\left(\frac{n-4}{z}+\frac{1}{2} \partial_{z} \chi-\frac{1}{f} \partial_{z} f\right) \partial_{z} \psi+\frac{r_{+}^{2} e^{\chi} q^{2} \phi^{2}}{f^{2} z^{4}} \psi-\frac{r_{+}^{2}}{z^{4}} \frac{1}{2 f} \frac{\partial V(\psi)}{\partial \psi}=0 \\
& \partial_{z} \eta=\frac{c_{1} e^{-\frac{1}{2} \chi}}{r_{+}^{n-3}} z^{n-4}-\frac{\alpha}{2} \partial_{z} \phi .
\end{aligned}
$$

In order to gain insight into the influence of the scalar filed on the background geometry, we solve the above system of differential equations perturbatively by expanding the unknown functions in the series in some small parameter $\varepsilon$. Because of the fact that the value of the scalar operator $\left\langle O_{i}\right\rangle$, where $i=-,+$, is small in the vicinity of the critical point, one introduces it as an expansion parameter $\varepsilon=<O_{i}>$ and expands the functions in question as follows:

$$
\begin{aligned}
f & =f_{0}+\varepsilon^{2} f_{2}+\varepsilon^{4} f_{4}+\ldots, \\
\chi & =\varepsilon^{2} \chi_{2}+\varepsilon^{4} \chi_{4}+\ldots, \\
\phi & =\phi_{0}+\varepsilon^{2} \phi_{2}+\varepsilon^{4} \phi_{4}+\ldots \\
\psi & =\varepsilon \psi_{1}+\varepsilon^{3} \psi_{3}+\ldots \\
\eta & =\eta_{0}+\varepsilon^{2} \eta_{2}+\varepsilon^{4} \eta_{4}+\ldots
\end{aligned}
$$

On this account, the chemical potential $\mu$ can be written in the form [48]

$$
\mu=\mu_{0}+\varepsilon^{2} \delta \mu
$$

where $\delta \mu>0$. It reveals that near the phase transition the order parameter may be described as a function of the chemical potential, i.e., $\varepsilon=\left(\left(\mu-\mu_{0}\right) / \delta \mu\right)^{1 / 2}$. The phase transition can take place when $\mu \rightarrow \mu_{0}$. Consequently, it can be observed that $\varepsilon$ approaches to zero and the critical value of the chemical potential is given by $\mu_{c}=\mu_{0}$.

At the zeroth order, the metric function will be provided by RN-AdS black hole spacetime, described in the preceding 
section. The new radial variable $z$ enables us to find the exact form of $\phi_{0}$ and $f_{0}$. Having in mind expressions in the nearby of the critical point and using equation (34), one concludes that

$$
\phi_{0}=\mu_{0}\left(1-z^{n-3}\right),
$$

where $\mu_{0}=\rho / r_{+}{ }^{n-3}$. On the other hand, at critical point when $\mu=\mu_{0}=\rho / r_{+c}{ }^{n-3}$, where $r_{+c}$ is the value of black hole event horizon radius at the critical point, we obtain

$$
\phi_{0}=\lambda r_{+c}\left(1-z^{n-3}\right),
$$

with $\lambda=\rho / r_{+c}^{n-2}$. The value $r_{+c}$, being value of the black hole event horizon at critical temperature, is bounded with the temperature in the sense that Hawking's black hole temperature depends on the value of the event horizon radius. It is provided by the relation

$$
T_{B H}=\left.\frac{1}{4 \pi} \partial_{r} g_{t t}\right|_{r=r_{\text {event hor }}} .
$$

Just the temperature of a black hole with event horizon radius value equal to $r_{+c}$ represents the critical temperature above which the condensation cannot take place.

Consequently, taking into account equation (32) and the relation (44) one achieves that $f_{0}$ is given by

$$
f_{0}=r_{+}^{2}\left[\frac{1}{L^{2} z^{2}}-\frac{z^{n-3}}{L^{2}}-z^{n-3}\left(1-z^{n-3}\right) \frac{2 \kappa^{2}}{n-2}\left(\frac{\tilde{\alpha}(n-3) \lambda^{2} r_{+c}^{2}}{r_{+}^{2}}+\frac{c_{1}^{2}}{(n-3) r_{+}^{2 n-4}}\right)\right],
$$

The lowest order corrections are solely determined by the $\psi$ field. The equation for this field (up to $\mathcal{O}\left(\varepsilon^{2}\right)$ terms) implies

$$
\partial_{z}^{2} \psi_{1}-\left(\frac{n-4}{z}-\frac{1}{f_{0}} \partial_{z} f_{0}\right) \partial_{z} \psi_{1}-\left(\frac{m^{2} r_{+}^{2}}{f_{0} z^{4}}-\frac{r_{+}^{2} q^{2} \phi_{0}^{2}}{z^{4} f_{0}^{2}}\right) \psi_{1}=0
$$

where one uses the fact that $\frac{\partial V(\psi)}{\partial \psi} \approx 2 m^{2} \varepsilon \psi_{1}+\mathcal{O}\left(\varepsilon^{3}\right)$. On the other hand, the asymptotic behaviour of the scalar field near the AdS boundary is provided by $\psi_{1}=\psi_{i} \frac{z^{\Delta_{i}}}{r_{+}}$, where $i= \pm$ and $\Delta_{i}=\frac{1}{2}\left[(n-1) \pm \sqrt{(n-1)^{2}+4 m^{2}}\right]$, while $\psi_{i}$ represents the expectation value of the adequate operator on the CFT side. Namely we choose as $\psi_{i}$ the expansion parameter $\left\langle O_{i}\right\rangle$. As the temperature leads to the critical one, scalar field $\psi$ approaches the limit which is even valid at $T=T_{c}$. Next, in order to match the behaviour at the boundary one defines $\psi_{1}$ in the form as follows:

$$
\psi_{1}=\frac{<O_{i}>}{r_{+}^{\Delta_{i}}} z^{\Delta_{i}} F(z)
$$

where we introduce a trial function $F(z)$ normalized to the following value $F(0)=1$. By virtue of the above relation we arrive at the equation

$$
\partial_{z}^{2} F+\left[\frac{2\left(\Delta_{i}+2\right)-n}{z}+\frac{\partial_{z} g}{g}\right] \partial_{z} F+\left[\frac{\Delta_{i}\left(\Delta_{i}-n+3\right)}{z^{2}}+\frac{\Delta_{i}}{z} \frac{\partial_{z} g}{g}-\frac{m^{2}}{z^{4} g}+\frac{q^{2} \lambda^{2} r_{+c}^{2}\left(1-z^{n-3}\right)^{2}}{r_{+}^{2} z^{4} g^{2}}\right] F=0 .
$$

To simplify the above equation we introduce a function $g$ related to $f_{0}$ by the following:

$$
f_{0}=r_{+}^{2}\left\{\frac{1}{L^{2} z^{2}}-\frac{z^{n-3}}{L^{2}}-z^{n-3}\left(1-z^{n-3}\right)\left[\frac{2 \kappa^{2}}{n-2}\left(\frac{\tilde{\alpha}(n-3) \lambda^{2} r_{+c}^{2}}{r_{+}^{2}}+\frac{c_{1}^{2}}{(n-3) r_{+}^{2 n-4}}\right)\right]\right\}=r_{+}^{2} g .
$$

In the next step we shall rewrite equation (49) in the form Sturm-Liouville form. Namely it can be done if one defines

$$
T=z^{2 \Delta_{i}+1}\left\{(n-2)\left(z^{1-n}-1\right)-L^{2} 2 \kappa^{2}\left[\frac{\tilde{\alpha}(n-3) \lambda^{2} r_{+c}^{2}}{r_{+}^{2}}+\frac{c_{1}^{2}}{(n-3) r_{+}^{2 n-4}}\right]\left(1-z^{n-3}\right)\right\},
$$

and after a little of algebra we find that we have left with

$$
\partial_{z}\left(T \partial_{z} F\right)+T\left\{\frac{\Delta_{i}}{z}\left[\frac{\Delta_{i}+3-n}{z}+\frac{\partial_{z} g}{g}\right]-\frac{m^{2}}{z^{4} g}+\lambda^{2} \frac{r_{+c}^{2} q^{2}\left(1-z^{n-3}\right)^{2}}{r_{+}^{2} z^{4} g^{2}}\right\} F=0 .
$$

The above equation is solved subject to the boundary condition $\partial_{z} F(0)=0$. 


\section{CRITICAL TEMPERATURE}

Now we refine our studies to the problem of finding the critical temperature below which the condensation of $\psi$ field can occur. An important notion bounded with the phase transition is the temperature connected with black hole in question. Using the definition of the Hawking temperature which for the line element in question

$$
T_{B H}=\left.\frac{1}{4 \pi} \partial_{r} f(r) e^{\frac{\chi(2)}{2}}\right|_{r=r_{+}},
$$

one can verify that, up to $\mathcal{O}(\varepsilon)$, it is provided by the relation

$$
T_{B H}=\frac{r_{+}}{4 \pi}\left\{\frac{n-1}{L^{2}}-\frac{2 \kappa^{2}}{n-2}(n-3)\left[\frac{\tilde{\alpha}(n-3) \lambda^{2} r_{+c}^{2}}{r_{+}^{2}}+\frac{c_{1}^{2}}{(n-3) r_{+}^{2 n-4}}\right]\right\},
$$

where $c_{1}$ is a constant, which can be partially restricted by the demand that we do not introduce any new parameters on the CFT side and the fact that the critical temperature scales with charge density in a typical way. In view of these postulates we calculate the black hole temperature at $r_{+}=r_{+c}$. It yields

$$
T_{B H}^{c}=\frac{1}{4 \pi}\left\{\frac{n-1}{L^{2}}-(n-3) \frac{2 \kappa^{2}}{n-2}\left[(n-3) \tilde{\alpha} \lambda^{2}+\tilde{c}^{2}\right]\right\}\left(\frac{\rho}{\lambda}\right)^{\frac{1}{n-2}}
$$

where we use the relation $c_{1}^{2}=\tilde{c}^{2}(n-3) r_{+}^{2 n-4}$.

On this account we shall find the critical temperature. Namely, having in mind equations (52) and (55), multiplying both sides of the relation (52) by the trial function $F(z)$ and integrating over the interval under consideration, we can specify $\lambda^{2}$ as a spectral parameter of the Sturm-Liouville eigenvalue problem [51]. On the other hand, the expression for estimating the minimum eigenvalue of $\lambda^{2}$ is provided by

$$
\lambda^{2} \leq \frac{\int_{0}^{1} d z\left[T\left(\partial_{z} F\right)^{2}-T U F^{2}\right]}{\int_{0}^{1} d z T V F^{2}},
$$

where the quantities $U$ and $V$ are defined by

$$
\begin{aligned}
& U=\frac{\Delta_{i}}{z}\left[\frac{\Delta_{i}+3-n}{z}+\frac{\partial_{z} g}{g}\right]-\frac{m^{2}}{z^{4} g} \\
& V=\frac{r_{+c}^{2} q^{2}\left(1-z^{n-3}\right)^{2}}{r_{+}^{2} z^{4} g^{2}}
\end{aligned}
$$

The validity of the use of the Sturm-Liouville method demands that our trial function must fulfill one of the prescribed sets of the boundary conditions [51]. Meanwhile beside this formal boundary conditions our test function should also be in the agreement with the physical boundary condition $\left(F(0)=1, \partial_{z} F(0)=0\right)$. Having this in mind we choose the following test function $F=1-a z^{2}+\frac{2}{3} a z^{3}$, where $a$ is a free parameter. The considered trial function satisfies all the aforementioned requirements. Integration of equation (56) enables us to obtain $\lambda^{2}$ as a function of a free parameter $a$ [34]. Further, one finds the minimum of this function and use it in the calculations of the critical temperature, the gravitational constant $\kappa$ is a backreacting parameter.

Having in mind equation (55), we remark that in comparison to the single $U(1)$ field case one has two new parameters being integration constants $\tilde{c}$ and the $\tilde{\alpha}=1-\frac{\alpha^{2}}{4}$, where $\alpha$ is a coupling constant between two gauge fields in the theory. Black hole temperature behaviour depends on whether $\tilde{c}^{2}$ is equal to $\left|(n-3) \tilde{\alpha} \lambda^{2}\right|$ or not.

Let us analyze the first case $\tilde{c}^{2} \neq\left|(n-3) \tilde{\alpha} \lambda^{2}\right|$. In this situation the exact value of $\tilde{c}$ is unimportant from the point of view of the qualitative behavior of the black hole temperature. The sign of the relation $(n-3) \tilde{\alpha} \lambda^{2}+\tilde{c}^{2}$ is important because of the $\alpha$-coupling dependence. Namely, if $\tilde{c}=0$ then for $\alpha<2$ it reaches a positive value, while $\alpha>2$ it is negative. Taking into account $\tilde{c} \neq 0$, one has that we need to consider the larger value of $\alpha$ to obtain a negative sign in the aforementioned expression.

In Table【we see that for a positive $\tilde{\alpha}$ and increasing value of the backreacting parameter $\kappa$, one obtains decreasing critical temperature. On the contrary, for the negative $\tilde{\alpha}$ the situation is quite opposite. For the completeness of reasoning we present also results for four-dimensional black hole in the Table II One can draw a conclusion that the change of the dimension of the underlying spacetime does not qualitatively change the phase transition temperature 


\begin{tabular}{|l|l|l|}
\hline \multicolumn{3}{|c|}{$n=5, \Delta=3, m^{2}=-3, L=1, q=1$} \\
\hline$\kappa$ & $\alpha=0.5(\tilde{\alpha}=0.94)$ & $\alpha=2.5(\tilde{\alpha}=-0.5625)$ \\
\hline$\kappa=0.0$ & $T_{B H}^{c}=0.1977 \rho^{\frac{1}{3}}$ & $T_{B H}^{c}=0.1977 \rho^{\frac{1}{3}}$ \\
\hline$\kappa=0.05$ & $T_{B H}^{c}=0.1926 \rho^{\frac{1}{3}}$ & $T_{B H}^{c}=0.2007 \rho^{\frac{1}{3}}$ \\
\hline$\kappa=0.1$ & $T_{B H}^{c}=0.1774 \rho^{\frac{1}{3}}$ & $T_{B H}^{c}=0.2099 \rho^{\frac{1}{3}}$ \\
\hline$\kappa=0.15$ & $T_{B H}^{c}=0.1533 \rho^{\frac{1}{3}}$ & $T_{B H}^{c}=0.2256 \rho^{\frac{1}{3}}$ \\
\hline$\kappa=0.2$ & $T_{B H}^{c}=0.1223 \rho^{\frac{1}{3}}$ & $T_{B H}^{c}=0.2484 \rho^{\frac{1}{3}}$ \\
\hline$\kappa=0.25$ & $T_{B H}^{c}=0.0882 \rho^{\frac{1}{3}}$ & $T_{B H}^{c}=0.2795 \rho^{\frac{1}{3}}$ \\
\hline$\kappa=0.3$ & $T_{B H}^{c}=0.0558 \rho^{\frac{1}{3}}$ & $T_{B H}^{c}=0.3201 \rho^{\frac{1}{3}}$ \\
\hline
\end{tabular}

TABLE I: The behavior of the critical temperature on the backreacting parameter $\kappa$ in the case of five-dimensional black hole, $\tilde{c}=0$.

\begin{tabular}{|l|l|l|}
\hline \multicolumn{3}{|c|}{$n=4, \Delta=2, m^{2}=-2, L=1, q=1$} \\
\hline$\kappa$ & $\alpha=0.5(\tilde{\alpha}=0.94)$ & $\alpha=2.5(\tilde{\alpha}=-0.5625)$ \\
\hline$\kappa=0.0$ & $T_{B H}^{c}=0.1182 \rho^{\frac{1}{2}}$ & $T_{B H}^{c}=0.1182 \rho^{\frac{1}{2}}$ \\
\hline$\kappa=0.05$ & $T_{B H}^{c}=0.1169 \rho^{\frac{1}{3}}$ & $T_{B H}^{c}=0.1190 \rho^{\frac{1}{3}}$ \\
\hline$\kappa=0.1$ & $T_{B H}^{c}=0.1128 \rho^{\frac{1}{2}}$ & $T_{B H}^{c}=0.1215 \rho^{\frac{1}{2}}$ \\
\hline$\kappa=0.15$ & $T_{B H}^{c}=0.1062 \rho^{\frac{1}{3}}$ & $T_{B H}^{c}=0.1256 \rho^{\frac{1}{3}}$ \\
\hline$\kappa=0.2$ & $T_{B H}^{c}=0.0974 \rho^{\frac{1}{2}}$ & $T_{B H}^{c}=0.1315 \rho^{\frac{1}{2}}$ \\
\hline$\kappa=0.25$ & $T_{B H}^{c}=0.0870 \rho^{\frac{1}{3}}$ & $T_{B H}^{c}=0.1394 \rho^{\frac{1}{3}}$ \\
\hline$\kappa=0.3$ & $T_{B H}^{c}=0.0756 \rho^{\frac{1}{2}}$ & $T_{B H}^{c}=0.1496 \rho^{\frac{1}{2}}$ \\
\hline
\end{tabular}

TABLE II: The behavior of the critical temperature on the backreacting parameter $\kappa$ in the case of four-dimensional black hole, $\tilde{c}=0$.

dependence on $\kappa$ and $\alpha$. To conclude, we may state that in the case $\tilde{c}^{2} \neq\left|(n-3) \tilde{\alpha} \lambda^{2}\right|$ one receives the two different types of behaviours of $T_{B H}^{c}(\kappa)$, i.e., for $(n-3) \tilde{\alpha} \lambda^{2}+\tilde{c}^{2}>0$ the temperature decreases with the increase of $\kappa$ and for the case when $(n-3) \tilde{\alpha} \lambda^{2}+\tilde{c}^{2}<0$, the temperature in question increases as $\kappa$ increases.

On the other hand, for $\tilde{c}^{2}=\left|(n-3) \tilde{\alpha} \lambda^{2}\right|$ we have only one type of behavior of $T_{B H}^{c}(\kappa)$, i.e., for the positive value of $\tilde{\alpha}$ when $\kappa$ increases, the temperature in question decreases. For a negative $\tilde{\alpha}$, the critical temperature is independent of $\kappa$ and equal to the $\kappa=0.0$ case. In Figs. 1 and 2 we depicted the aforementioned behaviour, i.e., the dependence of the critical temperature on $\kappa$ for four and five-dimensional spacetimes, having in mind the positive and negative values of the coupling constant $\tilde{\alpha}$. It should be also stressed that in the case of four-dimensional spacetime, when $\kappa$ is equal to zero our results are in a perfect agreement with the ones received in Ref. [9].

Summing it all up, it is worth mentioning that the behaviour responsible for the negative value of $\tilde{\alpha}$ is rather surprising, because of the fact that the condensed phase appears at high temperature, not at low as usual. We refer this action as a retrograde condensation [49]. The similar behaviour was observed in Ref.[50], where holographic superconductors were obtained from gauged supergravity theory with a complex scalar field.

\section{CONCLUSIONS}

In our paper we have used the variational method for the Sturm-Liouville eigenvalue problem for analytical studies of the holographic superconductor including backreactions of matter field sector on gravitational background. In the matter sector we assume a coupling between Maxwell gauge field and another $U(1)$-gauge field in order to mimic the possible dark matter sector influence on the critical temperature. One examined the cases of five and fourdimensional gravitational background as well as two possibilities of choosing the coupling constant $\tilde{\alpha}$. In the case when $\tilde{\alpha}>0$ one obtains that the bigger backreacting parameter we choose the smaller critical temperature we get. This behaviour was true for both five and four-dimensional holographic superconductors. On the contrary, when we take into account $\tilde{\alpha}<0$, the obtained results recall the retrograde condensation phenomenon [49]. Namely, the bigger $\kappa$ we imply the bigger critical temperature one achieves. The retrograde condensation was also observed in the case of gauged supergravity holographic superconductors [50]. In the limit of $\kappa=0$, when we have no backreaction, our 
analytical method gave the same value of the critical temperature as received by numerical methods in Ref. 9], i.e., $T_{B H}^{c}=0.1182 \rho^{\frac{1}{2}}$.

For the future researches, it will be interesting to examine the influence of the magnetic field on the dark matter sector holographic superconductor and the influence of the other gauge fields on superconductor parameters. We hope to prepend these problems elsewhere.

\section{Acknowledgments}

ŁN was supported by the Polish National Science Centre under doctoral scholarship number 2013/08/T/ST2/00122. MR was partially supported by the grant of the National Science Center 2011/01/B/ST2/00488.

[1] J.M.Maldacena, Adv. Theor. Math. Phys. 2, 231 (1998).

[2] E.Witten, Adv. Theor. Math. Phys. 2, 253 (1998),

S.S.Gubser, I.R.Klebanov, and A.M.Polyakov, Phys. Lett. B 428, 105 (1998).

[3] S.A.Hartnoll and P.Kovtun, Phys. Rev. D 76, 066001 (2007).

[4] S.A.Hartnoll, P.Kovtun, M.Muller, and S.Sachdev, Phys. Rev. B 76, 144502 (2007).

[5] S.A.Hartnoll, Class. Quantum Grav. 26, 224002 (2009), C.P.Herzog, J. Phys. A: Math. Gen. 42, 343001 (2009),

G.T.Horowitz, Introduction to Holographic Superconductors, hep-th 1002.1722 (2010).

[6] D.Mateos, Class. Quantum Grav. 24, S713 (2007).

[7] S.S.Gubser, Class. Quantum Grav. 22, 5121 (2005).

[8] S.S.Gubser, Phys. Rev. D 78, 065034 (2008).

[9] S.A.Hartnoll, C.P.Herzog, and G.T.Horowitz, Phys. Rev. Lett. 101, 031601 (2008).

[10] G.T.Horowitz and M.M.Roberts, Phys. Rev. D 78, 126008 (2008).

[11] S.A.Hartnoll, C.P.Herzog, and G.T.Horowitz, JHEP 12, 015 (2008).

[12] E.Nakano, W.-Y. Wen, Phys. Rev. D 78, 046004 (2008), K.Maeda, T.Okamura, Phys. Rev. D 78, 106006 (2008).

[13] M.Montull, A.Pomarol, P.J.Silva, Phys. Rev. Lett. 103, 091601 (2009), T.Albash, C.V.Johnson, Phys. Rev. D 80, 126009 (2009), K.Maeda, M.Natsuume, T.Okamura, Phys. Rev. D 81, 026002 (2010).

[14] M.Ammon, J.Erdmenger, V.Grass, P.Kerner, and A.O'Bannon, Phys. Lett. B 686, 192 (2010).

[15] R.G.Cai, Z.Y.Nie, and H.Q.Zhang, Phys. Rev. D 83, 066013 (2011).

[16] S.S.Gubser and A.Nellore, JHEP 04, 008 (2009).

[17] Y.Brihaye and B.Hartmann, Phys. Rev. D 81, 126008 (2010).

[18] G.T.Horowitz and B.Way, JHEP 11, 011 (2010).

[19] Y.Peng, Q.Y.Pan, and B.Wang, Phys. Lett. B 699, 383 (2011).

[20] A.Akhavan and M.Alishahiha, Phys. Rev. D 83, 086003 (2011).

[21] Y.Brihaye and B.Hartmann, Phys. Rev. D 83, 126008 (2011).

[22] R.Gregory, S.Kanno, and J.Soda, JHEP 10, 010 (2009).

[23] L.Barclay, R.Gregory, S.Kanno, and P.Sutcliffe, JHEP 12, 029 (2010).

[24] H.Zeng, Z.Fan, and Z.Ren, Phys. Rev. D 80, 066001 (2009).

[25] Q.Pan, B.Wang, E.Papantonopoulos, J.Oliviera, and A.Pavan, Phys. Rev. D 81, 106007 (2010).

[26] S.Kanno, Class. Quantum Grav. 28, 127001 (2011).

[27] F.Aprile and J.G.Russo, Phys. Rev. D 81, 026009 (2010).

[28] Y.Liu and Y.Sun, JHEP 07, 099 (2010).

[29] A.Salvio, Holographic superfluids and superconductors in dilaton gravity, hep-th 1207.3800 (2012).

[30] R.Cai and H.Zhang, Phys. Rev. D 81, 066003 (2010),

[31] F.Denef and S.A.Hartnoll, Landscape of superconducting membranes, hep-th 0901.1160 (2009), S.S.Gubser, C.P.Herzog, S.S.Pufu, and T.Tesileanu, Phys. Rev. Lett. 103, 141601 (2009), J.Gauntlett, J.Sonner, and T.Wiseman, ibid. 103, 151601 (2009).

[32] J.Jing and S.Chen, Phys. Lett. B 686, 68 (2010), R.Banerjee, S.Gangopadhyay,D.Roychowdhury, and A.Lala, Phys. Rev. D 87, 104001 (2013), Z.Zhao, Q.Pan, S.Chen, and J.Jing, Nucl. Phys. B 871, 98 (2013).

[33] J.Jing, Q.Pan, and S.Chen, JHEP 11, 05 (2011), J.Jing, Q.Pan, and B.Wang, Phys. Rev. D 84, 126020 (2011), J.Jing, B.Wang, Q.Pan, and S.Chen, ibid. 83, 066010 (2011), Z.Zhao, Q.Pan, S.Chen, and J.Jing, Nucl. Phys. B 871, 98 (2013). 
[34] G.Siopsis, J.Therrien, and S.Musiri, Class. Quantum Grav. 29, 085007 (2012),

H.B.Zheng, X.Gao, Y.Jiang, and H.S.Zong, JHEP 05, 002 (2011),

H.F.Li, R.G.Cai, and H.Q.Zhang, ibid. 04, 028 (2011),

J.L.Jing, Q.Y.Pan, and S.B.Chen, ibid. 11, 045 (2011),

H.-B. Zeng, X.Gao, Y.Jiang, and H.S.Zong, ibid. 05, 002 (2011),

Q.Pan, J.Jing, B.Wang, and S.Chen, ibid. 11, 088 (2011),

Q.Pan, J.Jing, B.Wang, and S.Chen, ibid. 06, 087 (2012),

S.Gangopadhyay and D.Roychowdhury, ibid. 05, 002 (2012),

S.Gangopadhyay and D.Roychowdhury, ibid. 05, 156 (2012).

[35] T.Shiromizu, S.Ohashi, and R.Suzuki, Phys. Rev. D 86, 064041 (2012).

[36] B.Bakon and M.Rogatko, Phys. Rev. D 87, 084065 (2013).

[37] S.Permutter, et al., Astrophys. J. 483, 565 (1997),

B.P.Schmidt, et al., ibid. 507, 46 (1998).

[38] C.L.Bennett et al., Astrophys. J. Suppl. 148, 1 (2003).

[39] D.J.Eisenstein et al. Astrophys. J. 633, 560 (2005).

[40] H.Davoudiasl, H.-S.Lee, I.Lewis, and W.J.Marciano, Phys. Rev. D 85, 115019 (2012), ibid. 88, 015022 (2013),

C.-F Chang, E.Ma, and T.-C.Yuan, Multilepton Higgs Deacays through the Dark Portal, hep-ph 1308.6071 (2014).

[41] T.Vachaspati and A.Achucarro, Phys. Reports 327, 347 (2000),

T.Vachaspati, Dark Strings, hep-th 0902.1764 (2009),

B.Hartmann and F.Arbabzadah, JHEP 07, 068 (2009),

Y.Brihaye and B.Hartmann, Phys. Rev. D 80, 123502 (2009).

[42] P.Jean et al., Astron. Astrophys. 407, L55 (2003).

[43] J.Chang et al., Nature (London) 456, 362 (2008).

[44] O.Adriani et al. (PAMELA Collaboration), Nature (London) 458, 607 (2009).

[45] G.W.Bennett et al., Phys. Rev. D 73, 072003 (2006).

[46] F.Bigazzi, A.L.Cotrone, D.Musso, N. Pinzani Fokeeva, and D.Seminara, JHEP 02, 078 (2012),

D.Musso, Minimal Model for an Unbalanced Holographic Superconductor, hep-th 1304.6118 (2013).

[47] K.Hashimoto and N.Iizuka, Impurities in Holography and Transport Coefficients, hep-th 1207.4643 (2012),

T.Ishii and S-J.Sin, Impurity Effect in a Holographic Superconductor, hep-th 1211.1798 (2013).

[48] C.P.Herzog, Phys. Rev. D 81, 126009 (2010).

[49] J.P.Kuenen, Commun. Phys. Lab. Univ. Leiden 4, (1892),

D.Katz and F.Kurata, Ind. Eng. Chem. 32, 817 (1940).

[50] F.Aprile, D.Roest, and J.G.Russo, JHEP 06, 040 (2011).

[51] A. D. Polyanin, V. F. Zaitsev, Handbook of Exact Solutions for Ordinary Differential Equations, Chapman\&Hall/CRC (2002). 


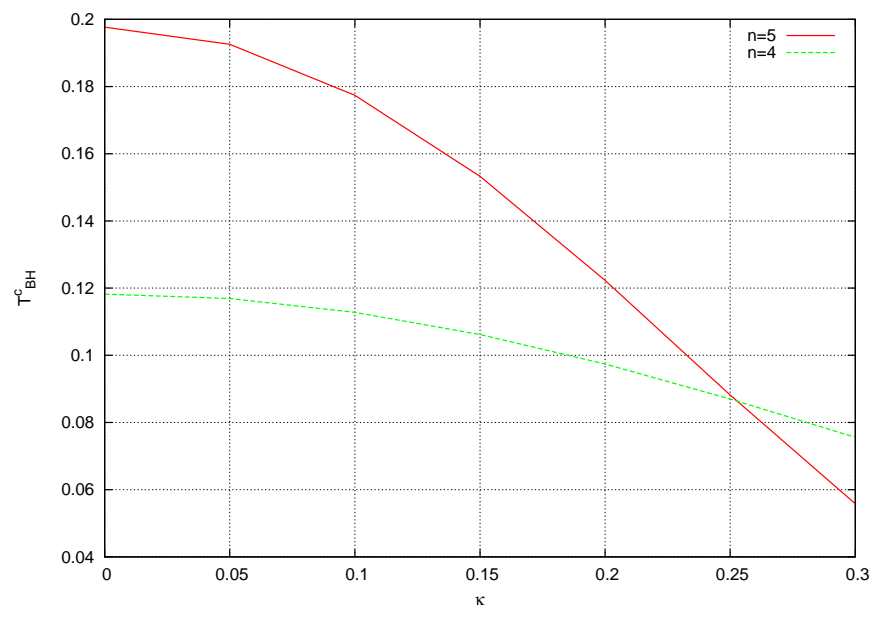

FIG. 1: (color online) The critical temperature as a function of $\kappa$ for the two different dimensions of spacetime. The parameters are chosen as $\tilde{\alpha}=0.94, L=1, q=1$, and $\Delta=3, m^{2}=-3$ for $n=5$ while $\Delta=2, m^{2}=-2$ for $n=4$.

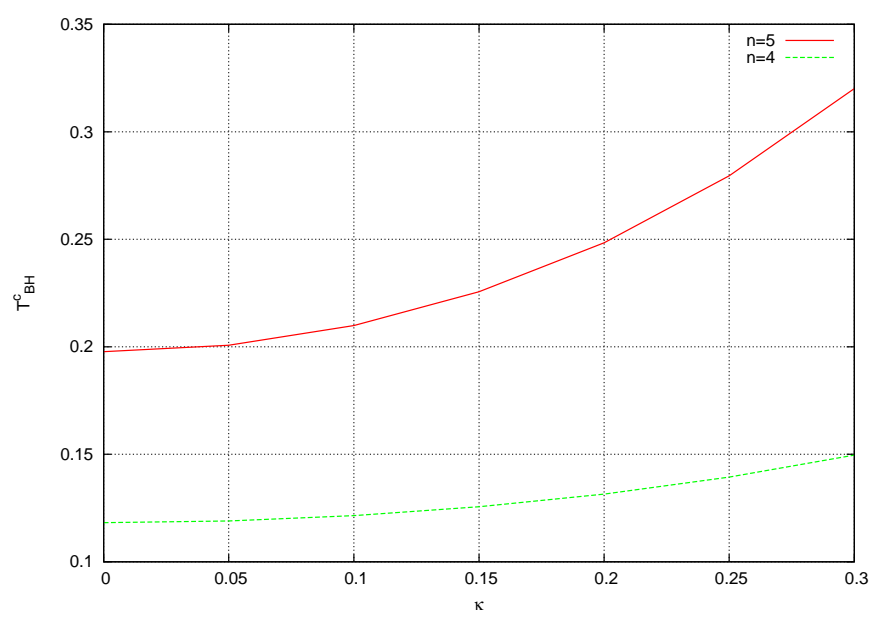

FIG. 2: (color online) The critical temperature as a function of $\kappa$ for two different dimensions of spacetime. The parameters are set as $\tilde{\alpha}=-0.5625, L=1, q=1$, and $\Delta=3, m^{2}=-3$ for $n=5$ while $\Delta=2, m^{2}=-2$ for $n=4$. 